\title{
Modelling of piezoelectric micro positioning system using direct mapping and numerical analysis for precision grinding process control
}

\author{
Y. Gao * and S. Tse
}

Department of Mechanical Engineering, Hong Kong University of Science and Technology, Clear Water Bay, Kowloon, Hong Kong SAR, China

E-mail: meygao@ust.hk* and meben@ust.hk

${ }^{*}$ Corresponding author

\begin{abstract}
For micro/nano positioning systems using piezoelectric actuators for precision grinding process control, sinusoidal command signals will be used and will give additional problems in comparison with the commonly used step signals due to the hysteresis effect, which require good modelling of the effect. To avoid discontinuity in obtaining the values of the piezoelectric constant, a method of direct mapping with polynomial fit has been proposed. Compared with the single polynomial approach, the model obtained was able to reduce the modelling error by $6.5 \%$. To further reduce the modelling error, a new method using direct mapping and numerical analysis is proposed while discontinuity is avoided. Theoretical and experimental studies are conducted and comparative studies are made. Compared with the single polynomial approach, it is able to reduce the modelling error to $7.3 \%$, which is $45.5 \%$ lower. Compared with the direct mapping with polynomial approach, the proposed method is able to reduce the modelling error by $41.7 \%$. For a typically fast piezoelectric micro/nano positioning system, hysteresis distortion is more significant towards modelling error than dynamic distortion. The proposed new approach should be useful in precision grinding process modelling and control.
\end{abstract}

Keywords: micro/nano positioning; piezoelectric hysteresis; direct mapping; numerical analysis; precision grinding process control.

Reference to this paper should be made as follows: Gao, Y. and Tse, S. (200x) 'Modelling of piezoelectric micro positioning system using direct mapping and numerical analysis for precision grinding process control', Int. J. Abrasive Technology, Vol. x, No. x, pp. xx-xx.

Biographical notes: Y. Gao is an associate professor of the Department of Mechanical Engineering, Hong Kong University of Science and Technology. His research interests include micro fabrication, abrasive technology, nanomachining, and in-process optical measurement.

$\mathrm{S}$. Tse is a PhD student of the Department of Mechanical Engineering, Hong Kong University of Science and Technology. He received his BSc degree in 1999 and his MSc degree in 2002. His research topic is precision control for surface grinding.

\section{Introduction}

It is known that, in surface grinding, a high speed workpiece table motion is involved. In order to achieve dynamic and active grinding process control for improved machining accuracy, the control commands will include substantial amount of high frequency signals. The signals may not be sufficiently implemented by a single actuating unit, typically the wheel infeed system, since the wheel system has a small frequency bandwidth for dynamic operations. A piezoelectric micro/nano positioning system utilizing a piezoelectric actuator may be used as an additional unit for providing small but fast control actions. The infeed system and the piezoelectric micro positioning system will work together under the composite control scheme (Gao, 1996; Gao, 2001; Gao et al., 


\section{2;.Gao et al., 2002).}

In order to investigate the characteristics of the piezoelectric micro/nano positioning system that operates over a range of frequencies and to obtain the response of the system for performance analysis, sinusoidal signals are utilized to be the command signals for analysis of the piezoelectric micro/nano positioning system.

For the system under the sinusoidal excitation, the hysteresis of the piezoelectric actuator has a significant effect (Fisher et al., 2004; Yang et al., 2000). Based on our previous studies (Gao et al., 2001-2006; Tse et al., 2005-2006), sinusoidal signals would give additional problems in comparison with the commonly used step signals. This requires further studies of the hysteresis characteristics of the piezoelectric actuator that is typically used in many micro/nano positioning systems.

In our previous investigations (Gao et al., 2004-2006; Tse et al., 2005-2006), models for the hysteresis behaviour and models for the transient response of the system under sinusoidal excitation were established (Gao et al., 2004-2006; Tse et al., 2005-2006). In one case, the hysteresis was modelled based on a single polynomial regression approach (Gao et al., 2004). An exhaustive search method was used to reduce the modelling error (Gao et al., 2004), which was up to approximately $15 \%$ (Gao et al., 2004).

Problems of discontinuity in obtaining values for the piezoelectric constant were experienced (Gao et al., 2005-2006; Tse et al., 2005-2006) in our previous studies. In order to avoid the problem of the kind and to reduce modelling error, direct mapping with polynomial fit was used (Tse et al., 2006). It was found that this approach was able to reduce the modelling error by $6.5 \%$ when compared with the result of the single polynomial approach and the problems in obtaining the values of the piezoelectric constant were avoided.

In order to further reduce the modelling error and solve the problems in obtaining the piezoelectric constant, a new method of direct mapping with numerical analysis is proposed. Theoretical and experimental studies are conducted and comparative studies are made for assessment of the proposed method.

\section{Piezoelectric micro positioning system model}

The mechanical model of the piezoelectric micro positioning system is shown in Fig. 1. Under the condition of no grinding force $F_{\mathrm{n}}(t)$, at the time instance $t$, the force generated by the piezoelectric translator $F_{\mathrm{pzt}}(t)$ is

$$
F_{\mathrm{pzt}}(t)=\left(k_{\mathrm{pzt}}+k_{\mathrm{s}}\right) y_{\mathrm{pzt}}(t),
$$

where $k_{\mathrm{pzt}}$ is the stiffness of the piezoelectric translator, $k_{\mathrm{s}}$ is the stiffness of the springs for preloading, $y_{\mathrm{pzt}}(t)$ is the transient displacement of the piezoelectric translator.

Based on the mechanical model (Fig. 1), the dynamic model of the system is given as

$$
\begin{aligned}
& \left(m_{\mathrm{pzt}}+m_{\mathrm{m}}\right) \ddot{y}(t)+\left(c_{\mathrm{pzt}}+c_{s}\right) \dot{y}(t)+\left(k_{\mathrm{pzt}}+k_{\mathrm{s}}\right) y(t), \\
& =F_{\mathrm{pzt}}(t)
\end{aligned}
$$

where $m_{\mathrm{pzt}}$ is the equivalent mass of the piezoelectric translator, $m_{\mathrm{m}}$ is the mass of the moving part in the micro positioning system, $c_{\mathrm{pzt}}$ is the equivalent damping coefficient of the piezoelectric translator, $c_{\mathrm{s}}$ is the equivalent damping coefficient of the springs, and $y(t)$ is the displacement response of the system (Fig. 1).

From Eq. (1) and Eq. (2), 


$$
\ddot{y}(t)+2 \zeta \omega_{\mathrm{n}} \dot{y}(t)+\omega_{\mathrm{n}}^{2} y(t)=\omega_{\mathrm{n}}^{2} y_{\mathrm{pzt}}(t),
$$

where

$$
\begin{gathered}
2 \zeta \omega_{\mathrm{n}}=\frac{c_{\mathrm{pzt}}+c_{\mathrm{s}}}{m_{\mathrm{pzt}}+m_{\mathrm{m}}}, \\
{\omega_{\mathrm{n}}}^{2}=\frac{k_{\mathrm{pzt}}+k_{\mathrm{s}}}{m_{\mathrm{pzt}}+m_{\mathrm{m}}},
\end{gathered}
$$

$\zeta$ is the damping factor, and $\omega_{\mathrm{n}}$ is the undamped natural frequency of the system.

In order to avoid the discontinuity problem in obtaining the values of piezoelectric constant $d_{33}$ (Gao et al., 2005-2006; Tse et al., 2005-2006), which requires special treatments, it was found that the displacement of the translator $y_{\mathrm{pzt}}(t)$ can be directly mapped with the input command signal $v_{\mathrm{d}}(t)$.

It is found that there is no need to determine a polynomial fit model for $y_{\mathrm{pzt}}\left(v_{\mathrm{d}}(t)\right)$ (Tse et al., 2006). Through the use of the experimentally obtained hysteresis loop (Fig. 2) and mapping, $y_{\mathrm{pzt}}(t)$ values can be obtained for given $v_{\mathrm{d}}(t)$ values.

Furthermore, the displacement response $y(t)$ (Eq. (3)) can be directly obtained by use of numerical analysis to solve the differential equation (Eq. (3)). It can be seen that the proposed new approach is further simplified (Tse et al., 2006).

For case study, two motion profiles for $v_{0}=5 \mathrm{~V}$, and $v_{0}=9 \mathrm{~V}$ are shown in Fig. 2 . The values of the displacement of the translator $y_{\mathrm{pzt}}(t)$ in Eq. (3) can be obtained directly from Fig. 2.

Figure 1 Mechanical model of the micro positioning system

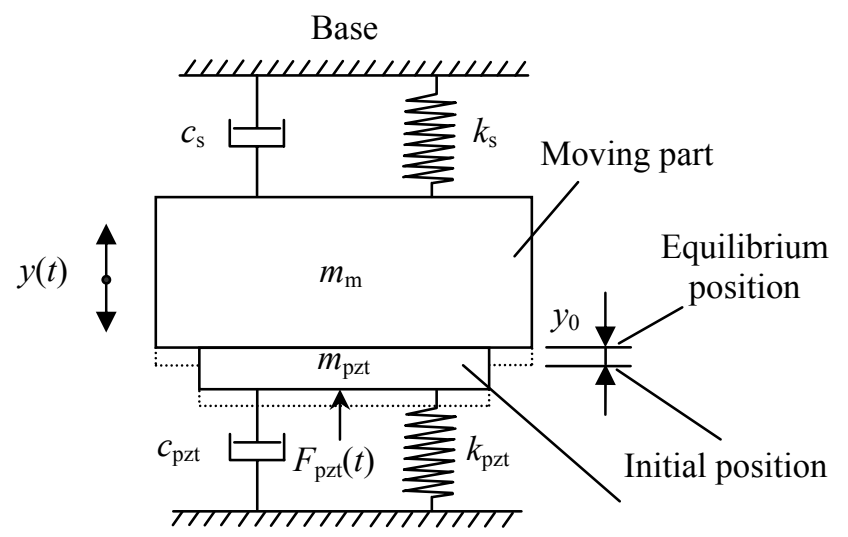

Base Equivalent mass of the piezoelectric translator 
Figure 2 Two motion profiles for $\mathrm{v}_{0}=5 \mathrm{~V}$ and $\mathrm{v}_{0}=9 \mathrm{~V}$, respectively

(a) $v_{0}=5 \mathrm{~V}$

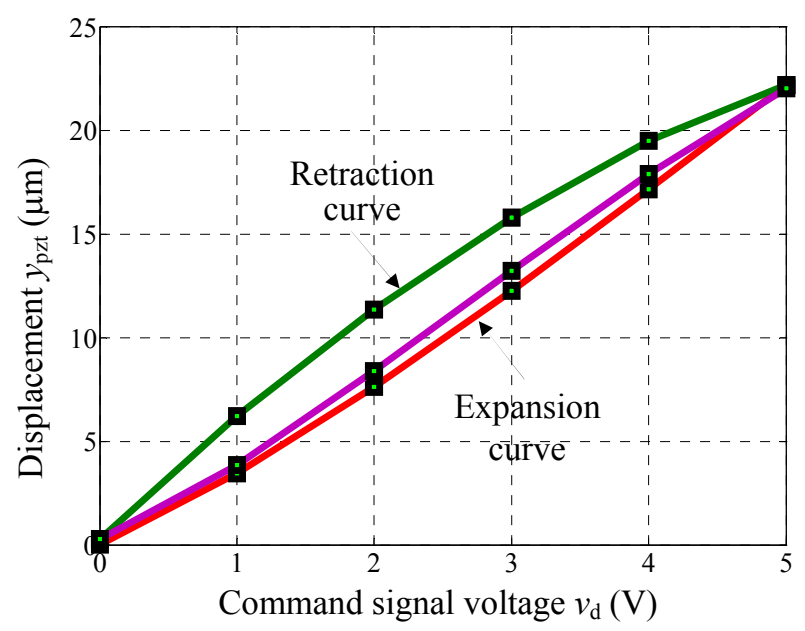

(b) $v_{0}=9 \mathrm{~V}$

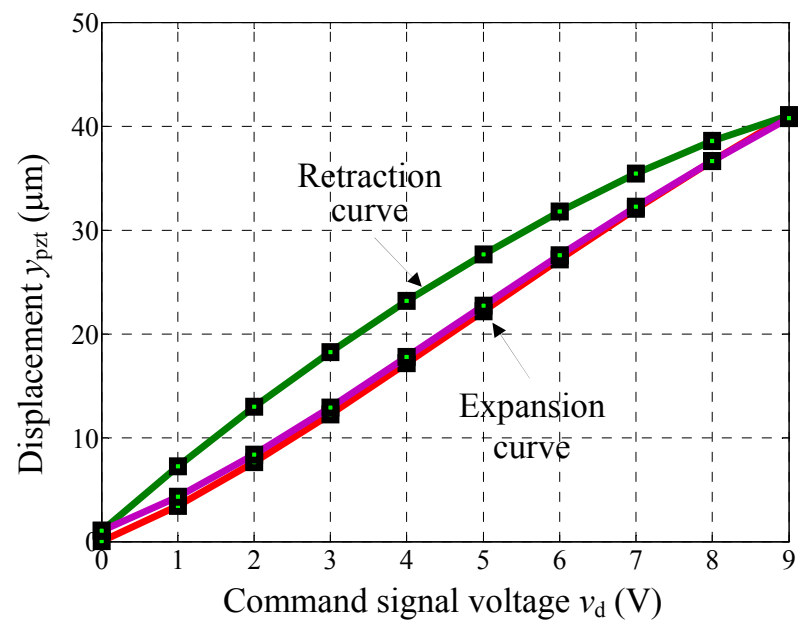

\section{Results and discussion}

3.1 Theoretical and experimental results

Theoretical displacement response $y(t)$ to a sinusoidal command signal $v_{\mathrm{d}}(t)$, where $v_{\mathrm{d}}(t)=v_{0}\left(1-\cos 2 \pi f_{\mathrm{e}} t\right) / 2$, can be determined using numerical analysis and Eq. (3). To obtain $y(t)$ (Eq. (3)), a number of parameters of the piezoelectric micro/nano positioning system 
are to be determined. In our previous work (Gao et al., 2001-2002), the damping factor of the micro/nano positioning table $\zeta$ was found equal to 0.0312 . The other parameters of the system were obtained from the specifications of the piezoelectric actuator PI P-840 and a number of tests. The equivalent mass of the translator $m_{\mathrm{pzt}}=4.3 \mathrm{~g}$, the mass of the moving part $m_{\mathrm{m}}=1.28 \mathrm{~kg}$, the stiffness of the piezoelectric translator $k_{\mathrm{pzt}}=17 \mathrm{~N} / \mu \mathrm{m}$, the stiffness of the springs for preloading $k_{\mathrm{s}}=1.2 * 10^{4} \mathrm{~N} / \mathrm{m}$, and as such $\omega_{\mathrm{n}}=3639.22 \mathrm{rad} / \mathrm{s}$.

To examine the proposed approach, the data obtained in our experimental tests (Gao et al., 2004-2006; Tse et al., 2005-2006) were used. In the experimental tests, the position (Fig. 1) was measured using a laser interferometer capable of dynamic measurement up to $5 \mathrm{kHz}$ in range and up to $1 \mathrm{~nm}$ in resolution.

Figures 3-6 show the experimental response $y_{\mathrm{e}}(t)$ and the theoretical response $y(t)$ for the excitation frequencies $f_{\mathrm{e}}=408.9 \mathrm{~Hz}, 334.5 \mathrm{~Hz}, 322.3 \mathrm{~Hz}$ and $247.8 \mathrm{~Hz}$, respectively. It can be seen that $v_{\mathrm{d}}(t)$ and $y_{\mathrm{e}}(t)$ are smooth while $y(t)$ is distorted. This is due to the distortion by the dynamics (Fig. 1) and the distortion by the hysteresis (Fig. 2). The dynamic distortion causes a difference in shape between $y_{\mathrm{pzt}}(t)$ and $y(t)$ (Eq. (3)). The hysteresis distortion causes a difference in shape between $v_{\mathrm{d}}(t)$ and $y_{\mathrm{pzt}}(t)$ or $F_{\mathrm{pzt}}(t)$ (Fig. 2 and Eq. (1)). Compared with the dynamic distortion, for a typically fast piezoelectric micro/nano positioning system (Fig. 1), hysteresis distortion (Fig. 2) is more significant towards the modeling error between $y(t)$ and $y_{\mathrm{e}}(t)$.

It is noted that $n_{\mathrm{c}}$ is the cycle count and the values of the excitation frequencies $f_{\mathrm{e}}$ were estimated by using a signal processing technique (Gao et al., 2005-2006; Tse et al., 2005-2006). The experimental and theoretical results are also shown in Table 1.

Figure 3 Result of $y_{\mathrm{e}}(t)$ and $y(t)$ for $n_{\mathrm{c}}=1$

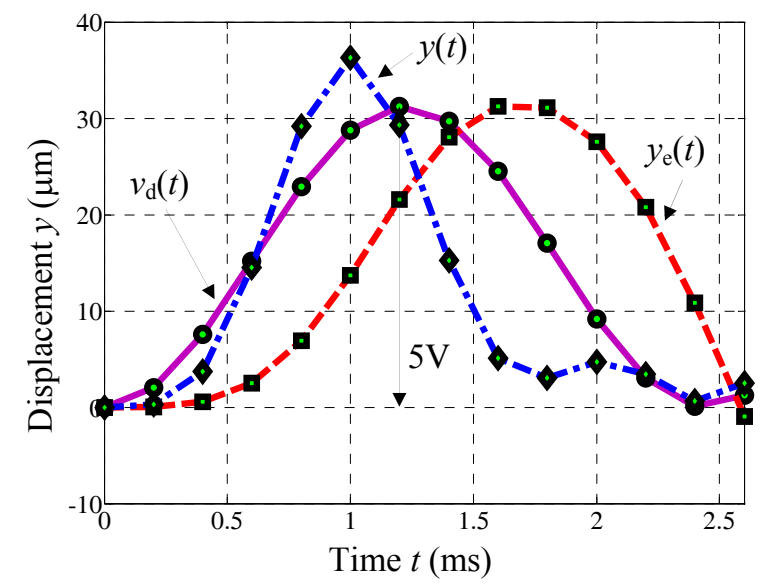


Figure 4 Result of $y_{\mathrm{e}}(t)$ and $y(t)$ for $n_{\mathrm{c}}=2$

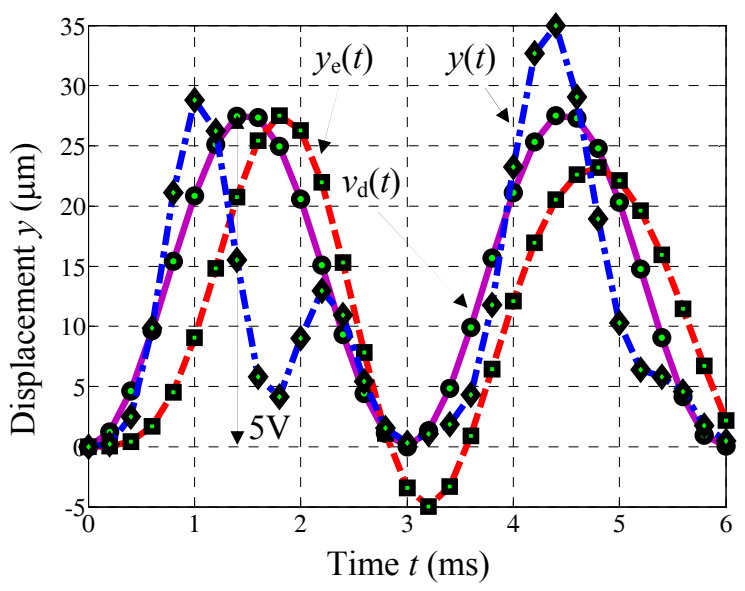

Figure 5 Result of $y_{\mathrm{e}}(t)$ and $y(t)$ for $v_{0}=5 \mathrm{~V}$

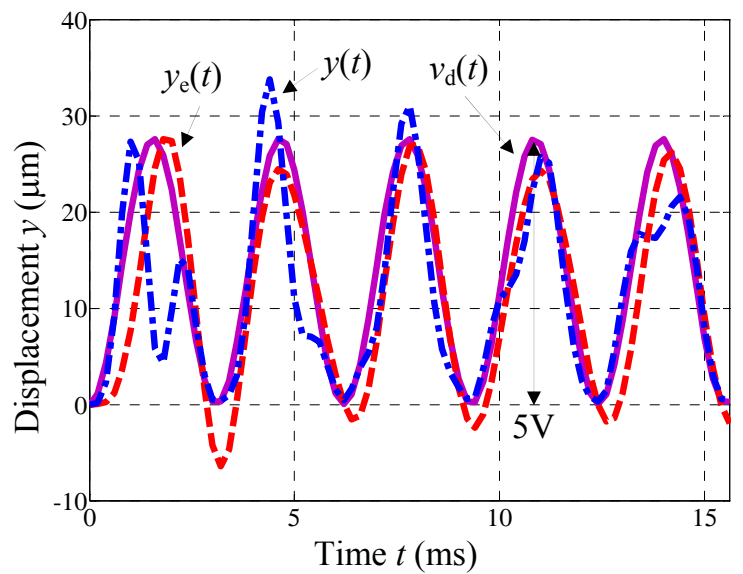


Figure 6 Result of $y_{\mathrm{e}}(t)$ and $y(t)$ for $v_{0}=9 \mathrm{~V}$

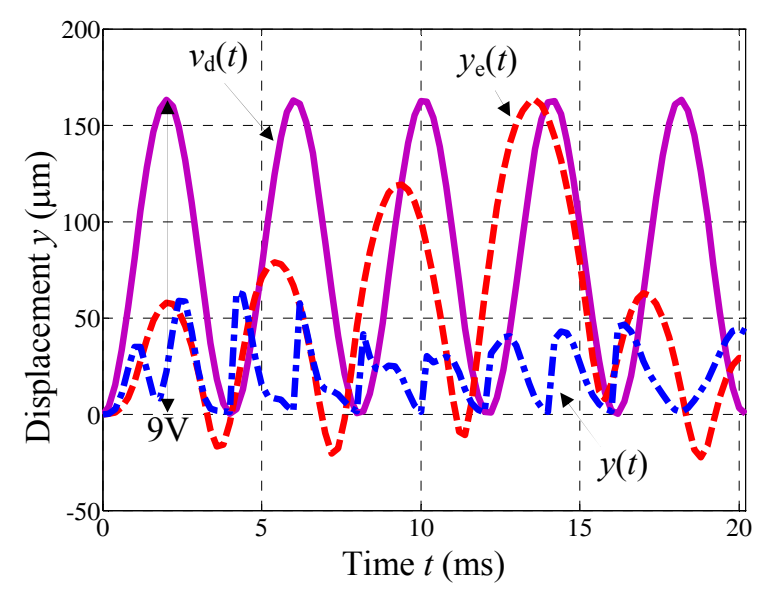

\subsection{Discussion}

To assess the proposed approach (Eq. (1)-(3)), the modelling error $e_{\mathrm{r}}$ will be compared. The peak values of the experimental response $y(t)_{\mathrm{emax}}$ and that of the theoretical response $y(t)_{\max }$ were examined (Figures 3-6 and Table 1).

Compared with the single polynomial approach (Gao et al., 2004-2005), noted as C1, the proposed new direct mapping with numerical analysis approach is able to reduce the modelling error $e_{\mathrm{r}}$ by $45.5 \%$ on average (Table 1), in addition to the advantage of possessing no discontinuity problem which was experienced (Gao et al., 2005-2006; Tse et al., 2005-2006).

Compared with the direct mapping with polynomial fit approach (Tse et al., 2006), noted as $\mathrm{C} 2$, the proposed new approach is able to reduce the modelling error $e_{\mathrm{r}}$ by $41.7 \%$ on average (Table 1).

For the proposed approach $(\mathrm{C} 3), e_{\mathrm{r}}=7.3 \%$ on average. The modelling error is smaller than the one using the model based on single polynomial and the model based on direct mapping with polynomial fit for a number of typical input voltages and frequencies (Gao et al., 2004; Tse et al., 2006). The results demonstrate the effectiveness of the proposed new method.

It is noted that the relative error $e_{\mathrm{r}}$ for C1-3 with $v_{0}=9 \mathrm{~V}$ and $n_{\mathrm{c}}=5$ was large (Table 1 ). This was due to a detachment problem similar to the one by a large step input command signal (Gao et al., 2001-2002).

\section{Conclusions}

Sinusoidal input signals would give additional problems in comparison with the commonly used step signals due to the hysteresis effect. A good modelling approach is needed. Using the proposed new method of direct mapping with numerical analysis, a modelling error of $7.3 \%$ was obtained. The proposed approach is advantageous since it is able to reduce the modelling error by $45.5 \%$ when compared with the single polynomial approach and by $41.7 \%$ when compared with the approach of direct mapping with 
polynomial fit. In addition, the need to obtain the values of the piezoelectric constant is removed. Compared with dynamic distortion, for a typically fast piezoelectric micro/nano positioning system, hysteresis distortion is typically more significant towards modelling error.

Table 1 Results of modeling error $e$ and relative error $e_{\mathrm{r}}$

\begin{tabular}{cccccccc}
\hline Case $^{\mathrm{a}}$ & $v_{0}(\mathrm{~V})$ & $n_{\mathrm{c}}$ & $f_{\mathrm{e}}(\mathrm{Hz})$ & $y(t)_{\mathrm{emax}}(\mu \mathrm{m})$ & $y(t)_{\max }(\mu \mathrm{m})$ & $e(\mu \mathrm{m})$ & $e_{r}(\%)$ \\
\hline $\mathrm{C} 1$ & 5 & 1 & 408.9 & 31.2607 & 34.6706 & 3.41 & 10.91 \\
$\mathrm{C} 2$ & 5 & 1 & 408.9 & 31.2607 & 34.6431 & 3.38 & 10.82 \\
$\mathrm{C} 3$ & 5 & 1 & 408.9 & 31.2607 & 36.2902 & 5.03 & 16.09 \\
$\mathrm{C} 1$ & 5 & 2 & 334.5 & 27.4849 & 31.9400 & 4.46 & 16.21 \\
$\mathrm{C} 2$ & 5 & 2 & 334.5 & 27.4849 & 31.3803 & 3.90 & 14.17 \\
$\mathrm{C} 3$ & 5 & 2 & 334.5 & 27.4849 & 28.7921 & 1.31 & 4.76 \\
$\mathrm{C} 1$ & 5 & 5 & 322.3 & 27.5795 & 31.1582 & 3.58 & 12.98 \\
$\mathrm{C} 2$ & 5 & 5 & 322.3 & 27.5795 & 31.0229 & 3.44 & 12.49 \\
$\mathrm{C} 3$ & 5 & 5 & 322.3 & 27.5795 & 27.3007 & 0.28 & 1.01 \\
$\mathrm{C} 1$ & 9 & 5 & 247.8 & 163.1977 & 37.9030 & 125 & 76.77 \\
$\mathrm{C} 2$ & 9 & 5 & 247.8 & 163.1977 & 47.3760 & 116 & 70.97 \\
$\mathrm{C} 3$ & 9 & 5 & 247.8 & 163.1977 & 42.8637 & 120 & 73.74 \\
\hline C1 - Single polynomial regression $\left[P_{i}\left(v_{\mathrm{d}}(t)\right), i=1\right](\mathrm{Gao}$ et al., $2004-2005)$ & \\
C2 - Direct mapping with polynomial fit & & & \\
C3 - Direct mapping with numerical analysis
\end{tabular}

\section{Acknowledgement}

This work has been supported by the Research Grants Council of Hong Kong SAR of China (Project no. 613206).

\section{References}

Fisher, R.S.L., Mclaughlin, E.A. and Robinson, H.C. (2004) 'Stress dependent behavior of $d_{33}$ in navy type iii and vi ceramics', J. Intelligent Material Systems and Structures, Vol. 15, pp. 549-566.

Yang, G., Liu, S.F., Ren, W. and Mukherjee, B.K. (2000) 'Uniaxial stress dependence of the piezoelectric properties of lead zirconate titanate ceramics', Proc. of SPIE, Vol. 3992, pp. 103-107.

Gao, Y. (1996) 'Composite control for the surface grinding process', Proc. of Int. Manufacturing Engineering Conf, pp. 157-159.

Gao, Y. (2001) 'Dynamics of a high performance workpiece table for active control during precision grinding', Proc. of ASPE 2001 Spring Topical Meeting, pp. 29-32.

Gao, Y., Zhang, D., Yu, C.W., Yang, Z. and Tse, S.F. (2002) 'Detachment modelling of a novel workpiece micro positioning table under preloaded hertz contact', Precision Engineering, Vol. 26, No. 1, pp. 83-92.

Gao, Y., Tse, S., Zhang, D. and Xu, X. (2002) 'Experimental validation of the dynamic models of a high performance workpiece table under preloaded hertzian contact', $J$. 
Materials Processing Technology, Vol. 129, pp. 485-489.

Gao, Y., Tse, S. and Chan, K. (2004) 'Modelling of sinusoidal excitation based on polynomial regression for micro positioning for grinding process control', Proc. of ISAAT 2004, pp. 365-37

Gao, Y. and Tse, S. (2005) 'An analysis of the discontinuity in the modelling of sinusoidal excitation for surface grinding control', Proc. of Euspen 2005, Vol. 2, pp. 377-380.

Gao, Y. and Tse, S. (2005) 'Modelling of piezoelectric actuator based nano-positioning system under sinusoidal excitation using multi-polynomial regression', Journal of Physics: Conference Series, IoP, ISMTII 2005, Vol. 13, pp. 98-101.

Tse, S. and Gao, Y. (2005) 'Modelling of piezoelectric actuator based nano-positioning system under sinusoidal excitation using multi-polynomial regression with $\mathrm{C}^{1}$ continuity', Key Engineering Materials, ASPEN 2005, Vol. 339, pp. 434-441.

Gao, Y. and Tse, S. (2005) 'Modelling of piezoelectric actuator based nano-positioning system under sinusoidal excitation using dual polynomial regression for surface grinding control', Proc. of ASPE 2005, ISBN 1-887706-39-9.

Tse, S. and Gao, Y. (2006) 'Modelling of micro positioning system using direct mapping and polynomial fit for precision grinding process control', Key Engineering Materials, ISAAT 2006, Vol. 329, pp. 791-796.

Gao, Y. and Tse, S. (2006) 'A study of the piezoelectric constant models for nanopositioning in precision machining', Proc. of ICFDM 2006, Vol. 2, pp. 139-144. 